\title{
MR Elastography Demonstrates Increased Brain Stiffness in Normal Pressure Hydrocephalus
}

\author{
(D) N. Fattahi, (D) A. Arani, (D)A. Perry, (D)F. Meyer, (D)A. Manduca, (D) K. Glaser, (D) M.L. Senjem, (D).L. Ehman, and (D). Huston
}

\begin{abstract}
BACKGROUND AND PURPOSE: Normal pressure hydrocephalus is a reversible neurologic disorder characterized by a triad of cognitive impairment, gait abnormality, and urinary incontinence that is commonly treated with ventriculoperitoneal shunt placement. However, multiple overlapping symptoms often make it difficult to differentiate normal pressure hydrocephalus from other types of dementia, and improved diagnostic techniques would help patient management. MR elastography is a novel diagnostic tool that could potentially identify patients with normal pressure hydrocephalus. The purpose of this study was to assess brain stiffness changes in patients with normal pressure hydrocephalus compared with age- and sex-matched cognitively healthy individuals.
\end{abstract}

MATERIALS AND METHODS: MR elastography was performed on 10 patients with normal pressure hydrocephalus and 21 age- and sex-matched volunteers with no known neurologic disorders. Image acquisition was conducted on a 3T MR imaging scanner. Shear waves with $60-\mathrm{Hz}$ vibration frequency were transmitted into the brain by a pillowlike passive driver. A novel postprocessing technique resistant to noise and edge artifacts was implemented to determine regional brain stiffness. The Wilcoxon rank sum test and linear regression were used for statistical analysis.

RESULTS: A significant increase in stiffness was observed in the cerebrum $(P=.001)$, occipital lobe $(P<.001)$, parietal lobe $(P=.001)$, and the temporal lobe $(P=.02)$ in the normal pressure hydrocephalus group compared with healthy controls. However, no significant difference was noted in other regions of the brain, including the frontal lobe $(P=.07)$, deep gray and white matter $(P=.43)$, or cerebellum $(P=.20)$.

CONCLUSIONS: This study demonstrates increased brain stiffness in patients with normal pressure hydrocephalus compared with ageand sex-matched healthy controls; these findings should motivate future studies investigating the use of MR elastography for this condition and the efficacy of shunt therapy.

ABBREVIATIONS: $\mathrm{NPH}=$ normal pressure hydrocephalus; $\mathrm{MRE}=$ MR elastography

N ormal pressure hydrocephalus $(\mathrm{NPH})$ is a potentially treatable condition characterized by cognitive impairment, gait abnormality, and urinary incontinence. A recent large epidemiologic study reported a dramatic increase in the prevalence of NPH after 80 years of age with only a minority of these cases undergoing treatment. ${ }^{1}$ With the progressive aging of our population, a continued increase in the prevalence of NPH can be expected in the future.

Received June 11, 2015; accepted after revision July 23.

From the Department of Radiology, Mayo Clinic, Rochester, Minnesota. This research was supported by the Theodore W. Batterman Family Foundation and R01 grant EB001981 (R.L.E.).

Please address correspondence to John Huston III, MD, Mayo Clinic 200 lst St SW \#W4, Rochester, MN 55905; e-mail: jhuston@mayo.edu

- Indicates open access to non-subscribers at www.ajnr.org

http://dx.doi.org/10.3174/ajnr.A4560
Multiple overlapping signs and symptoms among different types of cognitive impairment, such as NPH, Alzheimer disease, and vascular dementia ${ }^{2-4}$ create diagnostic challenges in these patients. These challenges will motivate the development and investigation of novel noninvasive imaging techniques to serve as diagnostic tools for identifying patients with NPH. The reversibility of neurologic symptoms after shunt tube placement supports the theory that deranged CSF circulation could play a role in NPH pathophysiology. Hypothetically, CSF accumulation may lead to local compression on the brain and can be the cause of this disorder. ${ }^{4}$ Therefore, recent studies have targeted hemodynamic and CSF circulation alterations and are investigating their role in intracranial pressure changes, which may be the cause of neurologic symptoms in $\mathrm{NPH}^{5-7}$

Although there is variability in the diagnosis of NPH, a highvolume lumbar tap with improvement of clinical symptoms as- 
Table 1: Patient distribution based on clinical manifestations and clinical response to treatment

\begin{tabular}{lc}
\hline \multicolumn{1}{c}{ Manifestation } & Distribution \\
\hline Clinical symptoms & $100 \%$ Gait disturbance \\
& $80 \%$ Cognitive problems \\
High volume CSF tap & $50 \%$ Urinary incontinence \\
& $100 \%$ Improvement of clinical \\
Response to shunt treatment & symptoms \\
& $90 \%$ Improvement of clinical \\
& symptoms \\
\hline
\end{tabular}

sessed by video studies obtained before and after the procedure is considered the most sensitive test for NPH at our institution. Several groups have shown that there is a strong correlation between surgical outcomes of shunt placement and a patient's response to a high-volume lumbar tap. ${ }^{5,8-13}$ However, several factors, including time intervals between CSF taps, CSF leaks, and the subjective nature of both the diagnosis and therapeutic response, may result in ambiguity in the interpretation of the results. Another disadvantage of a lumbar tap is the invasive nature of the test, with associated potential complications such as headache, infection, and CSF leakage. ${ }^{14}$ Neuroimaging currently has a prominent role in the diagnosis, assessment of therapy response, and monitoring of disease progression in patients with NPH. Disproportionate enlargement of cerebral ventricles, the Sylvian fissure, and the basal cistern in relation to the degree of cortical atrophy is the conventional MR imaging finding suggestive of NPH. ${ }^{15}$

MR elastography (MRE) is an MR imaging-based technique that noninvasively measures the mechanical properties of tissues in vivo. It has been shown that pressure changes that alter tissue elasticity are detectable by MRE. ${ }^{16,17}$ Therefore, the purpose of this study was to investigate possible brain stiffness changes in patients with NPH compared with age- and sex-matched healthy controls.

\section{MATERIALS AND METHODS}

This study was approved by our institutional review board, and all subjects provided informed written consent before recruitment and imaging.

\section{Subjects}

Ten patients with NPH ( 5 women and 5 men) with a mean age of 71 years (range, 67-79 years) were studied. All subjects were diagnosed with NPH on the basis of clinical symptoms and enlarged ventricles out of proportion to the size of the sulci (anatomic MR imaging) and improvement of symptoms following high-volume lumbar tap (Table 1). After undergoing MRE, all patients underwent CSF shunt surgery, and 9 of 10 patients experienced a remarkable improvement in symptoms after the procedure, which further supports the NPH diagnosis. Twenty-one cognitively healthy individuals without known neurologic disorders, 11 women and 10 men with a mean age 74 years (range, $67-80$ years), served as the healthy control group. The healthy control group was obtained from a subset of subjects previously recruited from a longitudinal study of aging who were imaged with MRE. ${ }^{18,19}$

\section{MRE}

Studies were performed on a 3T scanner (GE Healthcare, Milwaukee, Wisconsin) by using a single-shot, flow-compensated, spin-echo EPI pulse sequence. Shear waves were introduced into the brain from an active driver engine located outside the scan room through a soft pillowlike passive driver placed under the subject's head within an 8-channel receive-only head coil. $^{20}$ The frequency of vibration was $60 \mathrm{~Hz}$, and the MRE sequence was performed by using the following parameters: $\mathrm{TR} / \mathrm{TE}=3600 / 62 \mathrm{~ms} ; \mathrm{FOV}=24 \mathrm{~cm}$; bandwidth $= \pm 250 \mathrm{kHz}$; $72 \times 72$ imaging matrix reconstructed to $80 \times 80$; $3 \times$ parallel imaging acceleration; frequency encoding in the anteroposterior direction; 48 contiguous 3-mm-thick axial sections; one 4-G/cm 18.2-ms zero- and first-order moment nulled motionencoding gradient on each side of the refocusing radiofrequency pulse synchronized to the motion; motion encoding in the positive and negative $\mathrm{x}, \mathrm{y}$, and $\mathrm{z}$ directions; and 8 phase offsets sampled during 1 period of the $60-\mathrm{Hz}$ motion (the acquisition time was $<7$ minutes). The acquired images had 3-mm isotropic resolution.

\section{Image Processing}

We applied a previously published MRE postprocessing pipeline that masks out voxels with contributions from CSF, minimizes partial volume and edge effects, attempts to correct areas of low MR signal-to-noise ratio and low wave amplitude, and has previously been shown to have a coefficient of variation of $<1 \%$ for global brain stiffness and $<2 \%$ for the lobes of the brain and the cerebellum. ${ }^{21}$ Key features of the processing are the following: registration of the MRE data to a standard anatomic atlas, applying the vector curl operation on the first temporal harmonic of the acquired displacement field to reduce the effects of longitudinal waves and boundaries; adaptive filters for all derivative calculations to reduce partial volume effects at boundaries; and careful masking of the results from regional boundaries to minimize edge effects and contamination from CSF. The curl is a mathematic operation on a vector field (in this case, the displacement field with motion in all 3 directions). It is a combination of derivatives that quantifies rotational deformation (eg, shear waves), which is the only signal of interest in MRE, but suppresses all compressional deformation (eg, longitudinal waves). Last, an elastogram (map of stiffness, defined as wave speed squared times attenuation) was calculated by applying the direct inversion algorithm to the smoothed curl wave field. Additionally 2-phase offset images were acquired with zero motion amplitude to calculate the signal-to-noise ratio. We calculated the median stiffness of different ROIs in the brain. Each ROI was generated by applying a warped lobar atlas to a T1-weighted image. ROIs were then registered to the magnitude data obtained from the MRE as described previously. ${ }^{22}$ The full postprocessing pipeline to produce elastograms was applied separately to each ROI. The ROIs investigated in this study included the cerebrum (whole brain excluding cerebellum); frontal, temporal, parietal, occipital lobes; deep gray matter/ white matter (GM/WM) (insula, deep gray nuclei, and white matter tracts); and the cerebellum.

AJNR Am J Neuroradiol 37:462-67 Mar 2016 www.ajnr.org 


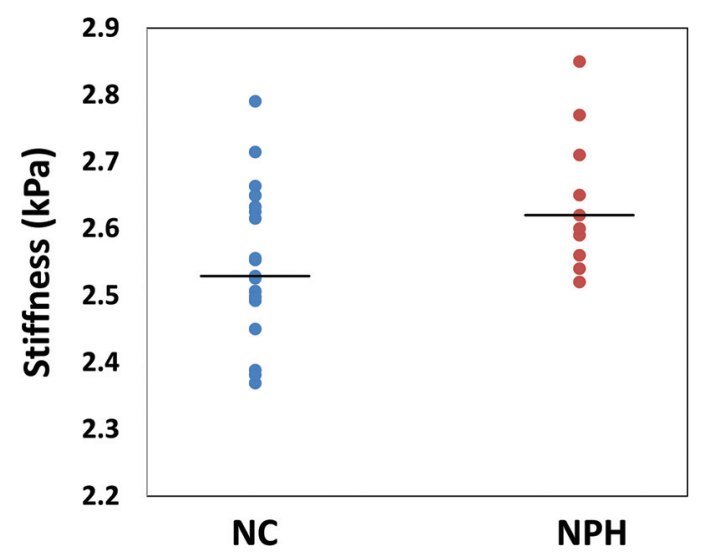

FIG 1. Summary of cerebral stiffness for the healthy controls (NC) and patients with normal pressure hydrocephalus. Lines represent the average stiffness for each group, and the circles represent the cerebral median stiffness for each individual patient.

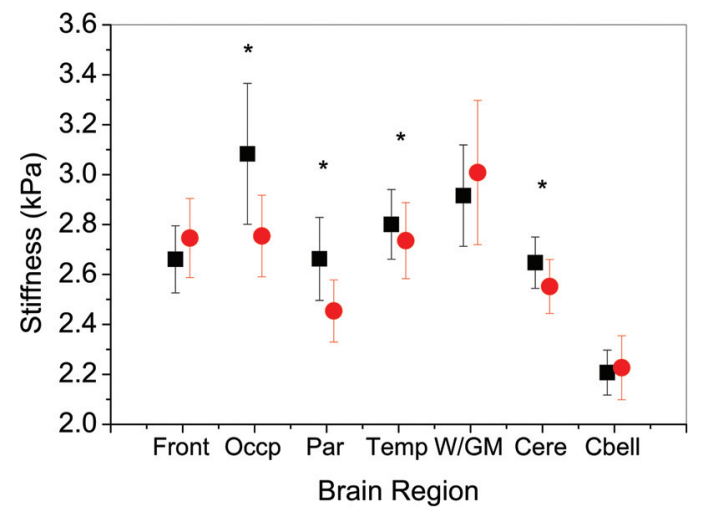

FIG 2. Mean of the median stiffness and SD of brain regions in patients with normal pressure hydrocephalus (black squares) and healthy controls (red circles). Asterisks indicate regions of significant stiffness difference between the NPH group and the healthy controls. Front indicates frontal lobe; Occp, occipital lobe; Par, parietal lobe; Temp, temporal lobe; W/GM, deep white/gray matter; Cere, cerebrum; Cbell, cerebellum.

\section{Statistical Analysis}

To compare brain tissue stiffness between patients with NPH and healthy controls, we used a Wilcoxon rank sum test; a $P$ value $<.05$ was considered statistically significant. A linear regression test was used to determine whether sex and age introduced a significant bias in the brain stiffness of the healthy control cohort.

\section{RESULTS}

The average median stiffness value of the cerebrum among patients with NPH was $2.64 \pm 0.1 \mathrm{kPa}$, which was significantly higher than the stiffness of the cerebrum in healthy controls $(2.55 \pm 0.1 \mathrm{kPa})(P=.001)$ (Fig 1). Significant increased stiffness was also observed in the occipital lobe $(P<.001)$, the parietal lobe $(P=.001)$, and the temporal lobe $(P=.02)$ in the NPH group (Figs 2 and 3). However, no significant difference was noted in other regions of the brain including the frontal lobe $(P=.07)$, deep GM/WM $(P=.43)$, or the cerebellum $(P=.20)$ (Table 2$)$. Brain stiffness of the healthy control group was fitted to a linear regression model to assess possible confounding factors of age and sex in the specific age range. A trend with age was found as in previous work, ${ }^{19}$ though in this age range it did not reach statis- tical significance $(P=.1)$. There was no significant linear relationship between sex and brain stiffness in our healthy control group $(P=.8)$.

\section{DISCUSSION}

In our study, patients with NPH demonstrated a significant increase of stiffness in the cerebrum and occipital, parietal, and temporal lobes compared with age- and sex-matched healthy controls. Although there is a significant difference between patients with NPH and healthy controls (Fig 1), there is too much overlap between the groups to be useful on an individual patient basis. Our findings stand in contrast to those of Streitberger et $\mathrm{al}^{23}$ who observed a significant decrease in the cerebral stiffness in patients with NPH compared with age- and sex-matched healthy controls. This discrepancy may be attributed to differences in the acquisition and postprocessing techniques. In our study, we reported 3-mm isotropic full-volume datasets segmented into anatomic lobes, while Streitberger et al took 3 adjacent 6-mm-thick sections and segmented them into global and periventricular regions. A considerable volume of the ROIs of Streitberger et al included the frontal lobes, which, in our work, showed a trend toward decreased stiffness in patients with NPH (though it did not reach significance). Other differences between the technique of Streitberger et al and ours, including differences in scanner hardware; processed data resolutions $(1.5 \times 1.5 \times 6 \mathrm{~mm}$ versus $3 \times 3 \times 3 \mathrm{~mm}$ ); TE ( 149 versus $\sim 60 \mathrm{~ms}$ ); number of time points (32 versus 8); and a processing approach that attempts to minimize CSF contamination and edge effects in this study, could potentially introduce SNR differences and stiffness estimation variations between the 2 techniques.

The physiology of NPH is dynamic, complex, and not wellunderstood, which makes it difficult to deduce a concrete mechanism behind the brain stiffening observed in this study. NPH generally refers to ventricular enlargement with normal opening pressure on lumbar puncture. However, overnight intermittent elevation of the intracranial pressure has been detected in patients with NPH, suggesting that increased intracranial pressure may play a role in the pathophysiology of $\mathrm{NPH} .{ }^{24}$ In addition, Alperin et $\mathrm{al}^{25}$ reported a linear pressure-volume relationship between the volumetric blood flow rate and intracranial pressure changes with a consistent elastance index. On the basis of these findings, ventricular dilation is thought to occur at the expense of the compressible compartment as interstitial and intracellular fluids are "squeezed" out of parenchymal pores. Brain tissue compression can cause tissues to move into the nonlinear elastic regime, causing stiffening, which would support our findings. Furthermore, corresponding cellular changes, such as a higher ratio of cytoskeletal matrix to interstitial and intracellular fluid has been reported, as well as a more compressed capillary and venous channel matrix, all of which could contribute to parenchymal stiffening and a loss of compliance. ${ }^{26,27}$

In this study, we found a significant increase in stiffness in the occipital, parietal, and temporal lobes, while lower elasticity values were measured in the frontal lobe and deep GM/WM areas in patients with NPH compared with healthy controls. Although these latter findings did not reach significance, it attracted our attention toward possible underlying changes that may contrib- 


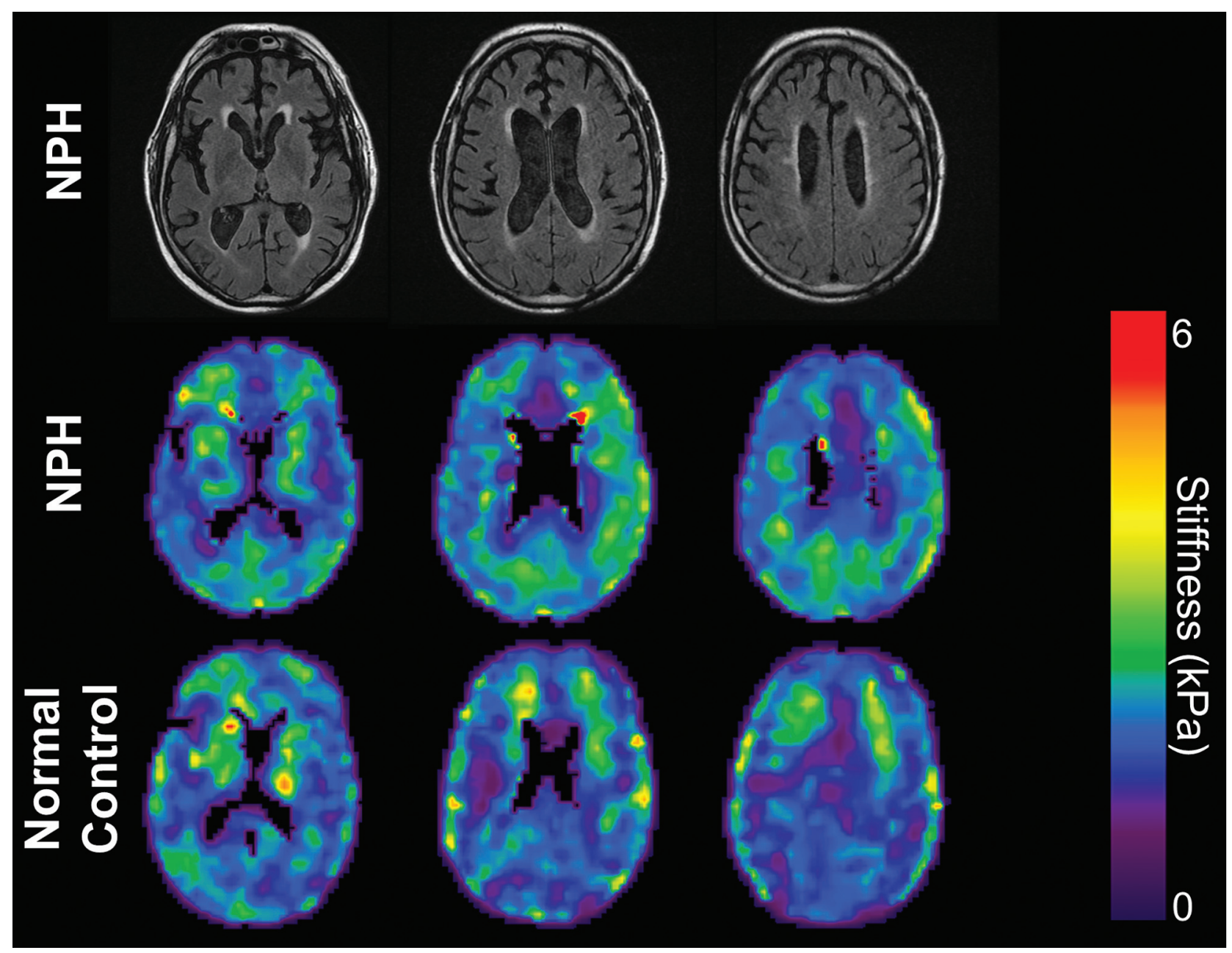

FIG 3. MRE image comparison of patients with NPH and healthy controls. T2 FLAIR images (top row) and MRE images (middle row) of a 67-year-old man with NPH. MRE of age- and sex-matched healthy controls (bottom row) shows increased stiffness in the patients with NPH compared with the healthy controls, especially in the parietal and occipital regions.

Table 2: MRE stiffness results

\begin{tabular}{lccc}
\hline & $\begin{array}{c}\text { Healthy Control } \\
\text { Brain Stiffness } \\
(\mathbf{k P a})\end{array}$ & $\begin{array}{c}\text { NPH Brain } \\
\text { Stiffness (kPa) }\end{array}$ & $\boldsymbol{P}$ Value \\
\hline Frontal lobe & $2.75 \pm 0.16$ & $2.66 \pm 0.13$ & .07 \\
Occipital lobe & $2.75 \pm 0.16$ & $3.08 \pm 0.28$ & $<.001$ \\
Parietal lobe & $2.45 \pm 0.12$ & $2.66 \pm 0.17$ & .001 \\
Temporal lobe & $2.73 \pm 0.15$ & $2.80 \pm 0.14$ & .02 \\
Deep GM/WM & $3.01 \pm 0.29$ & $2.91 \pm 0.20$ & .43 \\
Cerebrum & $2.55 \pm 0.11$ & $2.65 \pm 0.10$ & .001 \\
Cerebellum & $2.23 \pm 0.13$ & $2.21 \pm 0.09$ & .20 \\
\hline
\end{tabular}

ute to brain softening. Several imaging and histopathologic studies reported brain tissue degeneration beyond the paraventricular area, which was shown to be associated with acute or chronic hydrocephalus. ${ }^{28-30}$ Ziegelitz et $\mathrm{al}^{31}$ illustrated a significant reduction in cerebral blood flow, not only in the paraventricular area but also in the basal medial frontal cortex and deep gray matter in patients with NPH compared with healthy controls. In addition, they reported a positive correlation between decreased cerebral blood flow and the severity of clinical symptoms. Bugalho and Alves $^{32}$ suggested that predominant frontal lobe white matter lesions observed by T2-weighted MR imaging may be a cause of irreversible symptoms in NPH. Therefore, the combination of factors such as reduced blood flow, tissue degeneration, and the development of white matter lesions could result in tissue softening in some regions, which may be overcompensation for compressional stiffening effects.

Further evidence of these competing factors can be found in the diffusion-tensor imaging literature. DTI studies investigating microstructural changes in NPH have illustrated region-dependent neuronal changes throughout the brain. They have reported that neuronal integrity changes in the periventricular area, including the corticospinal tract, are consistent with changes secondary to mechanical compression and tend to reverse after shunt treatment. However, neuronal integrity changes in the frontal lobe white matter, corpus callosum, and deep gray matter are compatible with degenerative changes, which remain unchanged after treatment. 6,7,29,32-34 Furthermore, previous brain MRE studies on Alzheimer disease and multiple sclerosis have illustrated that decreased brain tissue stiffness is associated with neurodegenerative changes. ${ }^{20,33,34}$ Similarly, in this study, we hypothesized that the decreased stiffness in the frontal lobe and deep GM/WM may be attributed to the underlying neurodegenerative process resulting from NPH or reduced blood flow. ${ }^{13,24,35,36}$ In- 
creased pressure in these areas, producing elevated stiffness, due to ventricle enlargement may also contribute to a limited measurable reduction in stiffness. This theory is also compatible with clinical and neuroanatomic studies suggesting that cognitive impairment in NPH corresponds to irreversible changes in the deep GM/WM frontal subcortical areas, which is unlikely to improve after shunt treatment. ${ }^{37,38}$ Also, Freimann et al $^{39}$ have reported no significant change in stiffness after shunt tube placement in patients with NPH. These results should motivate future investigation into the relationship between regional brain stiffness and shunt placement outcomes.

\section{CONCLUSIONS}

Brain MRE of patients with NPH revealed increased brain tissue stiffness in the cerebrum and the occipital, parietal, and temporal lobes compared with age- and sex-matched healthy controls. Although not significant, decreased stiffness was observed in the frontal lobe and deep GM/WM of patients with NPH compared with healthy controls. This specific pattern of stiffness alteration will motivate future studies investigating the role of compressibility and degenerative changes in the development of NPH. In the future, MRE could potentially be implemented as a valuable diagnostic and prognostic tool for NPH and therapy response.

Disclosures: Nikoo Fattahi_RELATED: Grant: Theodore W. Batterman Family Foundation.* Armando Manduca—UNRELATED:Stock/Stock Options: stockholder in Resoundant, which works in the general area of MR elastography. Kevin GlaserRELATED: Grant: NIH R01 EB001981*; UNRELATED: Patents (planned, pending or issued): MR elastography*; Royalties: licensing of MR Elastography intellectual property; Stock/Stock Options: Resoundant. Matthew Senjem—RELATED: Grant: NIH.* Richard L. Ehman—RELATED: Grant: NIH (EB001981)*; UNRELATED: Board Membership: Resoundant (CEO, uncompensated); Grants/Grants Pending: Resoundant; Patents (planned, pending, or issued): R.L.E. and the Mayo Clinic have patents and intellectual property rights related to MRE; Royalties: Resoundant, Comments: Resoundant has licensed MRE technology from Mayo Clinic. Resoundant pays a licensing fee to Mayo Clinic, which is shared, in part, with R.L.E. and other inventors, pursuant to the Bayh-Dole act; Stock/Stock Options: Resoundant*; OTHER RELATIONSHIPS: The Mayo Clinic and R.L.E. have intellectual property rights and a financial interest in technologies used in this research. R.L.E. serves as CEO of Resoundant. This research was conducted under the oversight and in compliance with Mayo Clinic Conflict of Interest Review Board. John Huston-RELATED: Grant: NIH (NIH R01 grant EB001981), ${ }^{*}$ Theodore W. Batterman Family Foundation*; UNRELATED: Patents (planned, pending or issued): MR elastography brain patents planned; Stock/ Stock Options: Resoundant. *Money paid to the institution.

\section{REFERENCES}

1. Jaraj D, Rabiei K, Marlow T, et al. Prevalence of idiopathic normalpressure hydrocephalus. Neurology 2014;82:1449-54 CrossRef Medline

2. Leinonen V, Koivisto AM, Alafuzoff I, et al. Cortical brain biopsy in long-term prognostication of $\mathbf{4 6 8}$ patients with possible normal pressure hydrocephalus. Neurodegener Dis 2012;10:166-69 CrossRef Medline

3. Bech-Azeddine R, Høgh P, Juhler M, et al. Idiopathic normal-pressure hydrocephalus: clinical comorbidity correlated with cerebral biopsy findings and outcome of cerebrospinal fluid shunting. J Neurol Neurosurg Psychiatry 2007;78:157-61 CrossRef Medline

4. Chakravarty A. Unifying concept for Alzheimer's disease, vascular dementia and normal pressure hydrocephalus: a hypothesis. Med Hypotheses 2004;63:827-33 CrossRef Medline

5. Bateman GA. The pathophysiology of idiopathic normal pressure hydrocephalus: cerebral ischemia or altered venous hemodynamics? AJNR Am J Neuroradiol 2008;29:198-203 CrossRef Medline

6. Kamiya K, Hori M, Miyajima M, et al. Axon diameter and intraaxonal volume fraction of the corticospinal tract in idiopathic nor- mal pressure hydrocephalus measured by $\mathbf{q}$-space imaging. PLoS One 2014;9:e103842 CrossRef Medline

7. Klinge PM, Brooks DJ, Samii A, et al. Correlates of local cerebral blood flow (CBF) in normal pressure hydrocephalus patients before and after shunting: a retrospective analysis of $[(15) \mathrm{O}] \mathrm{H}(2) \mathrm{O}$ PET-CBF studies in 65 patients. Clin Neurol Neurosurg 2008;110: 369-75 CrossRef Medline

8. Wikkelso C, Andersson H, Blomstrand C, et al. The clinical effect of lumbar puncture in normal pressure hydrocephalus. J Neurol Neurosurg Psychiatry 1982;45:64-69 CrossRef Medline

9. Damasceno BP, Carelli EF, Honorato DC, et al. The predictive value of cerebrospinal fluid tap-test in normal pressure hydrocephalus. Arq Neuropsiquiatr 1997;55:179-85 CrossRef Medline

10. Marmarou A, Bergsneider M, Klinge $P$ et al. The value of supplemental prognostic tests for the preoperative assessment of idiopathic normal-pressure hydrocephalus. Neurosurgery 2005;57(3 suppl): S17-28; discussion ii-v Medline

11. Calcagni ML, Taralli S, Mangiola A, et al. Regional cerebral metabolic rate of glucose evaluation and clinical assessment in patients with idiopathic normal-pressure hydrocephalus before and after ventricular shunt placement: a prospective analysis. $\mathrm{Clin} \mathrm{Nucl} \mathrm{Med}$ 2013;38:426-31 CrossRef Medline

12. Lundin F, Ledin T, Wikkelsø C, et al. Postural function in idiopathic normal pressure hydrocephalus before and after shunt surgery: a controlled study using computerized dynamic posturography (EquiTest). Clin Neurol Neurosurg 2013;115:1626-31 CrossRef Medline

13. Lundin F, Ulander M, Svanborg E, et al. How active are patients with idiopathic normal pressure hydrocephalus and does activity improve after shunt surgery? A controlled actigraphic study. Clin Neurol Neurosurg 2013;115:192-96 CrossRef Medline

14. Kahlon B, Sundbärg G, Rehncrona S. Comparison between the lumbar infusion and CSF tap tests to predict outcome after shunt surgery in suspected normal pressure hydrocephalus. J Neurol Neurosurg Psychiatry 2002;73:721-26 CrossRef Medline

15. Kitagaki H, Ishii K, Yamaji S, et al. CSF spaces in idiopathic normal pressure hydrocephalus: morphology and volumetry. AJNR Am J Neuroradiol 1998;19:1277-84 Medline

16. Hirsch S, Beyer F, Guo J, et al. Compression-sensitive magnetic resonance elastography. Phys Med Biol 2013;58:5287-99 CrossRef Medline

17. Mousavi SR, Fehlner A, Streitberger KJ, et al. Measurement of in vivo cerebral volumetric strain induced by the Valsalva maneuver. J Biomech 2014;47:652-57 CrossRef

18. Roberts RO, Geda YE, Knopman DS, et al. The Mayo Clinic Study of Aging: design and sampling, participation, baseline measures and sample characteristics. Neuroepidemiology 2008;30:58-69 CrossRef Medline

19. Arani A, Murphy MC, Glaser KJ, et al. Measuring the effects of aging and sex on regional brain stiffness with MR elastography in healthy older adults. Neuroimage 2015;111:59-64 CrossRef Medline

20. Murphy MC, Huston J 3rd, Jack CR Jr, et al. Decreased brain stiffness in Alzheimer's disease determined by magnetic resonance elastography. J Magn Reson Imaging 2011;34:494-98 CrossRef Medline

21. Murphy MC, Huston J 3rd, Jack CR Jr, et al. Measuring the characteristic topography of brain stiffness with magnetic resonance elastography. PLoS One 2013;8:e81668 CrossRef Medline

22. Jack CR, Jr, Lowe VJ, Senjem ML, et al. 11C PiB and structural MRI provide complementary information in imaging of Alzheimer's disease and amnestic mild cognitive impairment. Brain 2008; 131 (pt 3):665-80 CrossRef Medline

23. Streitberger KJ, Wiener E, Hoffmann J, et al. In vivo viscoelastic properties of the brain in normal pressure hydrocephalus. $N M R$ Biomed 2011;24:385-92 CrossRef Medline

24. Eide PK, Brean A. Cerebrospinal fluid pulse pressure amplitude during lumbar infusion in idiopathic normal pressure hydroceph- 
alus can predict response to shunting. Cerebrospinal Fluid Res 2010; 7:5 CrossRef Medline

25. Alperin NJ, Lee SH, Loth F, et al. MR-intracranial pressure (ICP): a method to measure intracranial elastance and pressure noninvasively by means of MR imaging: baboon and human study. Radiology 2000;217:877-85 CrossRef Medline

26. Sklar FH, Diehl JT, Beyer CW Jr, et al. Brain elasticity changes with ventriculomegaly. J Neurosurg 1980;53:173-79 CrossRef Medline

27. Levine $\mathrm{DN}$. The pathogenesis of normal pressure hydrocephalus: a theoretical analysis. Bull Math Biol 1999;61:875-916 CrossRef Medline

28. Del Bigio MR, Cardoso ER, Halliday WC. Neuropathological changes in chronic adult hydrocephalus: cortical biopsies and autopsy findings. Can J Neurol Sci 1997;24:121-26 Medline

29. Tarnaris A, Toma AK, Pullen E, et al. Cognitive, biochemical, and imaging profile of patients suffering from idiopathic normal pressure hydrocephalus. Alzheimers Dement 2011;7:501-08 CrossRef Medline

30. Assaf Y, Ben-Sira L, Constantini S, et al. Diffusion tensor imaging in hydrocephalus: initial experience. AJNR Am J Neuroradiol 2006;27: 1717-24 Medline

31. Ziegelitz D, Starck G, Kristiansen D, et al. Cerebral perfusion measured by dynamic susceptibility contrast MRI is reduced in patients with idiopathic normal pressure hydrocephalus. J Magn Reson Imaging 2014;39:1533-42 CrossRef Medline

32. Bugalho P, Alves L. Normal-pressure hydrocephalus: white matter lesions correlate negatively with gait improvement after lumbar puncture. Clin Neurol Neurosurg 2007;109:774-78 CrossRef Medline

33. Murphy MC, Curran GL, Glaser KJ, et al. Magnetic resonance elastography of the brain in a mouse model of Alzheimer's disease: initial results. Magn Reson Imaging 2012;30:535-39 CrossRef Medline

34. Streitberger KJ, Sack I, Krefting D, et al. Brain viscoelasticity alteration in chronic-progressive multiple sclerosis. PLoS One 2012;7: e29888 CrossRef Medline

35. Qvarlander S, Lundkvist B, Koskinen LO et al. Pulsatility in CSF dynamics: pathophysiology of idiopathic normal pressure hydrocephalus. J Neurol Neurosurg Psychiatry 2013;84:735-41 CrossRef Medline

36. Hamlat A, Adn M, Sid-ahmed S, et al. Theoretical considerations on the pathophysiology of normal pressure hydrocephalus (NPH) and NPH-related dementia. Med Hypotheses 2006;67:115-23 CrossRef Medline

37. Poca MA, Mataró M, Matarín M, et al. Good outcome in patients with normal-pressure hydrocephalus and factors indicating poor prognosis. J Neurosurg 2005;103:455-63 CrossRef Medline

38. Kazui H. Cognitive impairment in patients with idiopathic normal pressure hydrocephalus [in Japanese]. Brain Nerve 2008;60:225-31 Medline

39. Freimann FB, Streitberger KJ, Klatt D, et al. Alteration of brain viscoelasticity after shunt treatment in normal pressure hydrocephalus. Neuroradiology 2012;54:189-96 CrossRef Medline 